\title{
Effects of response selection on the task repetition benefit in task switching
}

\author{
IRING KOCH and ANDREA M. PHILIPP \\ Max Planck Institute for Human Cognitive and Brain Sciences, Munich, Germany
}

\begin{abstract}
A task switch typically leads to worse performance than a repetition does. This shift cost can be reduced with sufficient task preparation time, but a residual cost usually remains. We propose that a large part of this residual cost is caused by an activation bias produced by response selection processes in the preceding trial. In our experiments, we manipulated response selection requirements using a go/no-go methodology. The residual shift cost disappeared after no-go trials, suggesting that response selection is crucial to establish an activation bias for the current category-response rules and that this bias persists into the next trial. A comparison with a go-only group confirmed this analysis by revealing no differences in preparatory strategy due to the inclusion of no-go trials. In addition, no-go trials had no significant effects on subsequent trials in a single-task experiment, suggesting that no-go trials are not coded as a task different from go trials and that there is no inhibition of the prepared task in a no-go trial. We thus conclude that a persisting activation bias of response rules plays a major role in task switching.
\end{abstract}

In many situations of everyday life, the environment changes quite rapidly, requiring flexible adaptation of behavior. The task-switching paradigm provides an experimental approach to study this flexibility in changing situations. Following the first experiments by Jersild (1927), there have been a variety of studies using that paradigm with diverse stimuli and tasks, but the key point is always the comparison of performance for repeating a task with that for a task switch. Typically, switching a task results in a higher reaction time (RT) and error rate than does a task repetition. This difference is often termed a shift cost (e.g., by Allport, Styles, \& Hsieh, 1994; Jersild, 1927; Meiran, 1996; Rogers \& Monsell, 1995).

Many studies have shown that shift cost can be reduced when participants are provided with a long task preparation time (e.g., Koch, 2003; Meiran, 1996; Rogers \& Monsell, 1995). However, the shift cost is usually not eliminated completely by preparation. Several explanations have been offered for this residual shift cost. For example, it has been attributed to the inability to finish reconfiguration before a stimulus is shown (Rogers \& Monsell, $1995)$ or to a failure to engage in intentional preparation in some of the trials (De Jong, 2000). Alternatively, Allport

This research was funded by Deutsche Forschungsgemeinschaft Grant KO 2045/4-1 to the first author. We thank Sander Los, Nachshon Meiran, and an anonymous reviewer for comments on a previous version of this article. We also thank Miriam Gade, Peter Keller, and Stefanie Schuch for helpful discussions concerning this work and Yi Zhang and Franziska Pöss for conducting parts of the experiments. Correspondence concerning this article should be addressed to I. Koch or A. M. Philipp, Max Planck Institute for Human Cognitive and Brain Sciences, Department of Psychology, Amalienstrasse 33, D-80799 Munich, Germany (e-mail: iring.koch@cbs.mpg.de, philipp@cbs.mpg.de). et al. (1994) attributed residual shift cost to proactive interference from the preceding trial. In the present study, we propose that a strong determinant of residual shift cost is the competition between task-specific response rules. We thus aim to explore the role that selecting a specific response rule plays for the residual shift cost.

Recently, an account stressing the role of response selection for proactive interference was proposed by Schuch and Koch (2003). They defined the term response selection rather broadly, as including the selection of the stimulus category and its mapping to the assigned manual response - in other words, the selection and application of a specific category-response (C-R) rule to a stimulus (e.g., "digit is odd - press the right key"). We will use the same broad definition of response selection in our study. Thus, our references to response selection will refer to the application of a task-specific C-R rule.

It is important to point out the difference between response selection and task preparation. We assume that in task preparation the next task goal is activated (e.g., to perform a parity task), and perhaps its stimulus categories as well (e.g., odd and even), but presumably not C-R rules (see Rubinstein, Meyer, \& Evans, 2001). Therefore, the role of response selection processes should be largely independent of the preparatory component in task switching (see Meiran, Chorev, \& Sapir, 2000) and thus would be crucial specifically for residual shift cost.

The importance of response selection in task switching was suggested by the findings of Schuch and Koch (2003). Their experiments mainly dealt with the question of whether the resolution of competition between C-R rules, which is assumed to take place at the point of response selection, results in the inhibition of irrelevant (i.e., competing) C-R rules. To do this, they used a paradigm 
recently introduced by Mayr and Keele (2000) to study the underlying process that Mayr and Keele called backward inhibition. To study the role of response selection for task inhibition, Schuch and Koch manipulated response selection by using a go/no-go methodology. They showed that inhibition was substantially reduced when a task was prepared but no response selection took place (i.e., after no-go trials), suggesting that inhibition depends to a large extent on response selection processes.

In the present study, we used the go/no-go methodology of Schuch and Koch (2003) to focus on the role of response selection for residual shift cost in task switching, whereas Schuch and Koch were mainly concerned with the effect of response selection on task inhibition.

The rationale for introducing no-go trials into a taskswitching experiment was to eliminate the need for response selection while keeping the task preparation component comparable with go trials. To do so, we used two numerical judgment tasks (greater vs. less than 5; odd vs. even) and converted a random sample of the trials into no-go trials so that the occurrence of a no-go trial was unpredictable. Each trial started with a task cue, indicating the upcoming task, followed by a variable task preparation time (see Meiran, 1996). The signal distinguishing between go and no-go trials (a high or low tone) was given simultaneously with stimulus onset. Hence, both types of trials had the same perceptual input, and participants had to engage in preparation with both types of trials, because no-go trials were unpredictable at the time of cue presentation. The only difference occurred at the point of stimulus presentation: Whereas participants had to select a response in a go trial, they did not need to do so in no-go trials. Thus, response selection was rendered unnecessary in no-go trials. (For a different way to use a go/no-go methodology in task switching, see Hoffmann, Kiesel, \& Sebald, 2003.)

As regards no-go trials, we assume that the go/no-go decision can be distinguished from response selection. The decision about whether a trial is go or no-go has to be made in every trial. Thus, the decision to respond or not to respond presumably is not equal to the decision to press the right or left key - that is, the go/no-go decision might increase overall task difficulty but should leave the competition of individual C-R rules on go trials largely unaffected. The effect of response selection, however, is caused by resolving interference between $\mathrm{C}-\mathrm{R}$ rules. Thus, the go/no-go decision and response selection should be distinguishable processes, and the decision not to respond should be differentiable from the selection and application of a C-R rule.

Schuch and Koch (2003, Experiments 1A and 1B) used the go/no-go methodology in a task-switching experiment with two tasks. They found a substantially reduced residual shift cost in trials following a no-go trial. However, it is important to note that this reduction of shift cost was mainly due to an increase in task-repeat RT rather than a decrease in task-switch RT. If no-go trials reduced shift cost in the following trial, one would actually have expected task-switch RT to decrease after no-go. However, task-repeat RT increased to the level of task-switch RT, but there was no substantial effect on task-switch RT. Schuch and Koch explained this finding by attributing it to a general increase in RT after no-go trials, presumably due to a priming of the no-go decision, producing a response delay in both switch and repetition trials. However, because Schuch and Koch focused on inhibition of C-R rules, they did not further pursue this no-go effect on shift cost but turned to the inhibition effect in three further experiments.

In the present study, we present new data that suggest an interpretation of the no-go effect on residual shift cost as a loss of the task repetition benefit when a task is only prepared but not executed. We prefer stressing the notion of a task repetition benefit because we assume that not only persisting inhibition but also persisting activation of $\mathrm{C}-\mathrm{R}$ rules plays a role in residual shift cost.

\section{Overview of Experiments}

We report three experiments that tested the role of response-related persisting activation using either a go/ no-go or go/double-press methodology. Specifically, we examined several theoretical alternatives that might account for no-go effects in task switching. The first alternative is that preventing response selection in some trials (i.e., no-go trials) generally discourages task preparation processes in task switching. Reduced task preparation in all trials leads to a situation in which a task is neither prepared nor activated after a no-go trial. In contrast, in go trials that follow other go trials there would also be no task preparation, but the just-executed task would provide the benefit of a persisting activation bias. Consequently, a shift cost would appear in go trials following go trials, but RTs for both tasks would be equally slow after no-go trials. In order to examine this alternative account, in Experiment 1 we compared performance of a no-go group with that of a go-only control group who participated in a similar experiment without no-go trials. Moreover, comparing performance of the no-go and goonly groups should show the effect of the additional go/ no-go decision, which was required only for the no-go group and not for the go-only group.

Experiment 2 was run to disentangle the roles of response selection and response execution. In this experiment, no-go trials were replaced with double-press trials in which an unspecific response execution was necessary rather than a task-specific response selection. A comparison of the results from Experiments 1 and 2 addressed the question of whether the relevant process that is rendered unnecessary in no-go trials is indeed the selection of a task-specific $\mathrm{C}-\mathrm{R}$ rule against competing rules.

A more theoretical alternative to our persisting activation bias account is that no-go trials might not be perceived as "normal" trials without response selection and execution but as a different kind of "task" altogether. If so, this would abolish any repetition benefit, because trials after no-go trials would always be de facto switch tri- 
als. A variant of this alternative would be to assume that no-go trials lead to "self-inhibition" of the current task, and thus also to impaired performance of task repetitions following no-go trials. Finally, one could assume that no-go trials lead to priming of the no-go decision, which then slows down performance generally after no-go trials (see Schuch \& Koch, 2003). These three alternative accounts appear to make basically the same predictions, albeit for different reasons. If these accounts were correct, though, we would also predict a general slowing after no-go trials in a single-task, two-choice situation. We tested this prediction in Experiment 3 by examining the effects of no-go trials in a single-task condition.

As we will demonstrate in the General Discussion section, the results of our experiments do not support the alternative accounts, but reinforce the proposal that a persisting activation bias of $\mathrm{C}-\mathrm{R}$ rules plays a major role in task switching.

\section{EXPERIMENT 1}

Experiment 1 was run to test whether task preparation is generally impaired when participants occasionally experience no-go trials, for which preparation is useless. To test this task preparation hypothesis, we converted $25 \%$ of the trials in this experiment to no-go trials for a no-go group, whereas a go-only group performed the experiment without no-go trials. In order to manipulate preparation time, we varied the length of the cue-stimulus interval (CSI). An effect of CSI on performance would indicate that participants prepared for the upcoming task. Finding that task preparation effects are the same for both groups would reinforce the notion that no-go trials affect response selection but not preparation processes. We predicted that RT levels should be generally higher in the no-go group than in the go-only group because an additional go/no-go decision was only required for the no-go group.

\section{Method}

Participants. Thirty-two participants ( 22 female, 10 male; mean age $=25.2$ years) were evenly assigned to the experimental groups (go-only vs. no-go). They received $€ 7$ for participation.

Stimuli and Tasks. Stimuli and tasks were identical for the two experimental groups, except for the presence or absence of no-go trials. Stimuli consisted of the digits $1-9$, excluding the number 5 . The participants had to decide whether a digit was greater or less than 5 (magnitude task) or whether it was odd or even (parity task). Stimuli were presented one at a time, in white, within a frame at the center of a black screen (15-in. monitor) connected to an IBM-compatible PC. The frame, which served as the task cue, was also white and had either the shape of a diamond (width/height of $5.3 \mathrm{~cm}$ ), indicating the magnitude task, or a square (width/height of $3.8 \mathrm{~cm}$ ), indicating the parity task. The digits were $1 \mathrm{~cm}$ high and approximately $0.5 \mathrm{~cm}$ wide. The viewing distance was $60 \mathrm{~cm}$.

Two easily discriminable tones $(200 \mathrm{~Hz}$ vs. $600 \mathrm{~Hz})$ indicated whether a response was required (go trial) or not (no-go trial). Responses were made manually on an external keyboard with response keys for the right and left index fingers. Response keys measured $1.2 \times 1.7 \mathrm{~cm}$ and were separated by $3.8 \mathrm{~cm}$.

Procedure. The experiment was run in a single session of $\sim 45 \mathrm{~min}$. Instructions emphasizing speed as well as accuracy were given both on the monitor and orally. The participants were informed about the tasks and the response keys, and an instruction sheet concerning the category-response key mapping was placed in front of them throughout the experiment. The four possible mappings were counterbalanced across participants.

For the no-go group, a sequence of four tones (high-low-highlow) was given before practice blocks started in order to demonstrate the difference between the two tones. Two practice blocks were run with 8 (go-only group) or 10 (no-go group) trials each. The different numbers of trials per block were used to keep the number of go trials in practice blocks comparable. One practice block had a short CSI (100 msec), and the other, a long CSI (1,000 msec). The experiment itself consisted of eight blocks of 96 trials each. Before each block, the participants were informed about the CSI in the block. Blocks with short and long CSIs alternated within the experiment; CSI duration in the first block was counterbalanced across participants.

Response-stimulus interval was held constant at $1,600 \mathrm{msec}$, with a response-cue interval (RCI) of either 600 or $1,500 \mathrm{msec}$ and a CSI of either 1,000 or $100 \mathrm{msec}$ (i.e., RCI 600/CSI 1,000 vs. RCI $1,500 / \mathrm{CSI} 100)$. Each trial began immediately after the response to the previous trial. A trial started with a black screen for the RCI duration, followed by a cue for the CSI duration. After this variable preparation time, the stimulus was presented in the middle of the cue frame along with a simultaneous high tone (indicating a go trial) or low tone (indicating a no-go trial). Although the go-only group experienced only go trials, the tone was played for them as well in order to maximize comparability across groups.

In the no-go group, the occurrence of no-go trials was unpredictable for the participants. In contrast to Schuch and Koch's (2003) Experiment 1A, for our no-go group the repetition of a no-go trial was possible. In this way, we eliminated the certainty of a no-go trial always being followed by a go trial. Since there were fewer no-go than go trials, the number of possible no-go repetitions was restricted to one in order not to produce too severe a loss in the number of observations. In go trials, pressing a response key removed cue and stimulus from the screen. In no-go trials, the stimulus was visible for $1,000 \mathrm{msec}$ and then vanished. This period was used to ensure that the total length of go and no-go trials was approximately comparable. The participants always received visual error feedback for $500 \mathrm{msec}$ when they pressed the wrong key (in German, Falsche Taste) in a go trial or when they pressed a key in a no-go trial (Keine Taste drücken, meaning "Do not press any key").

The sequence of trials was controlled for equal numbers of trials with each task and category within a task (odd, even, smaller, greater); for number of go and no-go trials; for task transition (repeat vs. switch); for task in trial $n-2$ (i.e., for equal numbers of repetitions and switches from trial $n-2$ to trial $n-1)$; and for response repetition. Repetition of a stimulus in consecutive trials was avoided. In addition, if a stimulus was presented for one task, this stimulus could not be repeated the next time the task occurred.

Design. Task transition (repeat vs. switch) and CSI (100 vs. $1,000 \mathrm{msec}$ ) were within-subjects independent variables. Group (goonly vs. no-go) was a between-subjects variable. In the no-go group, previous trial type (go vs. no-go in trial $n-1$ ) was a third withinsubjects variable. RT and error percentage for go trials were measured as dependent variables. Significance was tested at $\alpha=.05$.

\section{Results and Discussion}

The first two trials of each block were discarded from analysis, as were trials with RTs above 3,000 $\mathrm{msec}(0.4 \%$ in the go-only and $1.0 \%$ in the no-go group). For RT analysis, only correct trials preceded by at least one other correct trial were included. The go-only group had a mean error rate of $7.3 \%$. In the no-go group, there were $5.1 \%$ errors in go trials (and $5.4 \%$ responses in no-go trials). RT and error data are shown in Table 1. 
Sequential effects of no-go trials (within subjects). First we report the sequential effects of no-go trials, before we present the data of the between-subjects comparison. We ran an analysis of variance (ANOVA) for the no-go group only with task transition, CSI, and previous trial type (i.e., go vs. no-go in trial $n-1$ ) as withinsubjects variables. The ANOVA revealed a significant effect of previous trial type $\left[F(1,15)=24.8, M S_{\mathrm{e}}=\right.$ $19,486.6, p<.001$ ], indicating that RT in go trials was shorter after a go trial $(992 \mathrm{msec})$ than after a no-go trial $(1,115 \mathrm{msec})$. The relevance of this effect is explained by referring to its interaction with task transition. We predicted that residual shift cost would be reduced after no-go trials, because after no-go trials there is no activation bias favoring task repetitions. The interaction of previous trial type and task transition was indeed significant $\left[F(1,15)=43.2, M S_{\mathrm{e}}=6,048.4, p<.001\right]$, and shift

Table 1A

Experiment 1: Mean Reaction Time (in Milliseconds) of Go Trials as a Function of Task Transition (Repeat vs. Switch), Type of Trial in $n-1$ (Go vs. No-Go), Cue-Stimulus Interval (CSI) in Trial $n$ (100 vs. 1,000 msec), and Group (No-Go vs. Go-Only)

\begin{tabular}{|c|c|c|c|c|c|c|}
\hline & \multicolumn{4}{|c|}{ Task Transition } & \multirow{2}{*}{\multicolumn{2}{|c|}{$\begin{array}{c}\text { Task Repetition } \\
\text { Benefit }\end{array}$}} \\
\hline & \multicolumn{2}{|c|}{ Repeat } & \multicolumn{2}{|c|}{ Switch } & & \\
\hline & $M$ & $S D$ & $M$ & $S D$ & $M$ & $S D$ \\
\hline \multicolumn{7}{|c|}{ No-Go Group } \\
\hline \multicolumn{7}{|l|}{ Go in $n-1$} \\
\hline CSI 100 & 974 & 219 & 1,184 & 283 & $210^{*}$ & 140 \\
\hline CSI 1,000 & 818 & 156 & 990 & 220 & $172^{*}$ & 101 \\
\hline \multicolumn{7}{|l|}{ No-go in $n-1$} \\
\hline CSI 100 & 1,199 & 260 & 1,225 & 295 & 26 & 118 \\
\hline CSI 1,000 & 1,020 & 196 & 1,014 & 226 & -6 & 144 \\
\hline \multicolumn{7}{|c|}{ Go-Only Group } \\
\hline Go in $n-1$ & & & & & & \\
\hline CSI 100 & 763 & 145 & 916 & 182 & $153^{*}$ & 69 \\
\hline CSI 1,000 & 581 & 105 & 681 & 147 & $100^{*}$ & 77 \\
\hline
\end{tabular}

${ }^{*} p<.05$ (task repetition benefit was tested with one-tailed $t$ tests).

Table 1B

Experiment 1: Mean Error Percentage of Go Trials as a Function of Task Transition (Repeat vs. Switch), Type of Trial in $n-1$ (Go vs. No-Go), Cue-Stimulus Interval (CSI) in Trial $n$ (100 vs. 1,000 msec), and Group (No-Go vs. Go-Only)

\begin{tabular}{|c|c|c|c|c|c|c|}
\hline & \multicolumn{4}{|c|}{ Task Transition } & \multirow{2}{*}{\multicolumn{2}{|c|}{$\begin{array}{c}\text { Task Repetition } \\
\text { Benefit }\end{array}$}} \\
\hline & \multicolumn{2}{|c|}{ Repeat } & \multicolumn{2}{|c|}{ Switch } & & \\
\hline & $M$ & $S D$ & $M$ & $S D$ & $M$ & $S D$ \\
\hline \multicolumn{7}{|c|}{ No-Go Group } \\
\hline Go in $n-1$ & & & & & & \\
\hline CSI 100 & 4.0 & 4.4 & 6.4 & 3.7 & $2.4^{*}$ & 4.1 \\
\hline CSI 1,000 & 4.0 & 3.1 & 5.1 & 4.2 & 1.1 & 3.0 \\
\hline \multicolumn{7}{|l|}{ No-go in $n-1$} \\
\hline CSI 100 & 4.8 & 3.6 & 6.8 & 6.4 & 2.0 & 6.5 \\
\hline CSI 1,000 & 6.3 & 6.3 & 5.4 & 5.1 & -0.9 & 6.0 \\
\hline \multicolumn{7}{|c|}{ Go-Only Group } \\
\hline Go in $n-1$ & & & & & & \\
\hline CSI 100 & 6.5 & 4.4 & 9.3 & 5.0 & $2.8^{*}$ & 2.5 \\
\hline CSI 1,000 & 5.9 & 3.7 & 7.7 & 4.1 & $1.8^{*}$ & 2.0 \\
\hline
\end{tabular}

${ }^{*} p<.05$ (task repetition benefit was tested with one-tailed $t$ tests). cost was markedly reduced after no-go trials (from $191 \mathrm{msec}$ to $10 \mathrm{msec}$ ).

Moreover, the main effects of task transition $[F(1,15)=$ 23.8, $\left.M S_{\mathrm{e}}=13,455.3, p<.001\right]$ and $\operatorname{CSI}[F(1,15)=31.4$, $\left.M S_{\mathrm{e}}=34,822.7, p<.001\right]$ were also significant. That is, RT was shorter in task-repeat than in task-switch trials and was reduced after a long preparation time. However, the interaction of task transition and CSI $[F(1,15)=1.5$, $\left.M S_{\mathrm{e}}=6,571.3, p=.25\right]$ was not significant. In order to understand this nonsignificance, it is important to consider that go trials following both go and no-go trials are included in this analysis: For go trials following no-go trials, we did not expect to find a large shift cost, leaving little opportunity for a substantial reduction of shift cost with a long CSI. However, the three-way interaction was also not significant $(F<1)$, because there was a small, nonsignificant preparatory benefit even after no-go trials. To clarify this pattern, we tested post hoc the preparatory reduction of shift cost (i.e., the task transition $\times$ CSI interaction) for go and no-go trials individually. As expected, a significant 38 -msec reduction was found for go trials following go trials ( $p=.031$, one-tailed $t$ test), but there was no significant interaction for go trials following no-go trials ( $p>.24$, one-tailed $t$ test). Thus, after go trials we found that shift cost was indeed significantly reduced with long preparation time.

For error percentages, a three-way ANOVA with the variables task transition, CSI, and previous trial type revealed a significant effect only for task transition $\left[F(1,15)=6.8, M S_{\mathrm{e}}=628.7, p=.020\right]$. No other main effect or interaction yielded significance (all $F$ s $<2.8$, all $p \mathrm{~s}>.115$ ). In general, though, the error data showed a pattern similar to that found in the RT data.

In sum, the data clearly suggest that the task was prepared in all trials, as shown by the CSI main effect and the preparatory reduction of shift cost after go trials. However, there was no response selection in no-go trials, and therefore no need for the resolution of competition between C-R rules. Alternatively, in no-go trials there might even be no competition of $\mathrm{C}-\mathrm{R}$ rules in the first place. Either way, no-go trials elicited no activation bias favoring task repetitions, resulting in higher RTs than were found for task repetitions after go trials.

The effect of introducing no-go trials (between subjects). A comparison between the two experimental groups should establish whether the introduction of no-go trials had a general effect on performance in sequences of go trials in each group. To obtain comparability across groups, for the no-go group only those go trials preceded by at least one other go trial (go/go) were included in this analysis.

A three-way ANOVA with RT as the dependent variable was performed, with the within-subjects independent variables task transition and CSI and the betweensubjects variable group. This analysis yielded significant effects of task transition $\left[F(1,30)=95.8, M S_{\mathrm{e}}=8,375.8\right.$, $p<.001]$ and CSI $\left[F(1,30)=84.9, M S_{\mathrm{e}}=13,865.7, p<\right.$ $.001]$, as well as a significant interaction between the 
variables $\left[F(1,30)=9.2, M S_{\mathrm{e}}=1,766.1, p=.005\right]$. For sequences of go trials, the data replicated the pattern commonly observed in task-switching experiments with an external task-cuing paradigm (e.g., Meiran, 1996): RTs in switch trials were higher than in repeat trials (943 vs. $784 \mathrm{msec}$ ), and a long CSI reduced mean RT (from 959 to $768 \mathrm{msec}$ ) as well as shift cost (from $182 \mathrm{msec}$ to $136 \mathrm{msec}$ ).

The effect of group was significant $[F(1,30)=17.6$, $\left.M S_{\mathrm{e}}=119,696.9, p<.001\right]$, indicating that the general RT level was higher in the no-go group than in the goonly group (992 vs. $735 \mathrm{msec}$ ). Thus, the introduction of no-go trials generally increased RT in go trials. This result can be explained partially by the additional, time-consuming go/no-go decision, and partially by a more conservative response criterion in the no-go group (see below). Yet, the group variable did not significantly interact with any other variable, suggesting that the introduction of no-go trials did not affect the size of the CSI effect or the interaction of task transition and CSI in go trial sequences $\left(F_{\mathrm{S}}<1\right.$ for the corresponding interactions). Hence, the comparison of performance in both groups showed that the introduction of no-go trials does not generally impair task preparation. In light of the questions of whether and how the additional decision load introduced by no-go trials affects performance, we found a general slowing due to the extra decision load, but this general slowing does not seem to be related to task-specific preparation.

Note, however, that a data trend does suggest an interaction of task transition and group $\left[F(1,30)=3.9, M S_{\mathrm{e}}=\right.$ $8,375.8, p=.057]$. Descriptively, shift cost was higher in the no-go group (191 msec) than in the go-only group $(127 \mathrm{msec})$. However, the error cost was higher in the goonly group than in the no-go group (see below). Therefore, it appears prudent not to interpret these nonsignificant differences in shift cost across groups.

Error rates were subjected to the same analysis as RT data. A three-way ANOVA comparing sequences of go trials (go/go) between groups revealed a significant effect of task transition $\left[F(1,30)=29.0, M S_{\mathrm{e}}=450.8, p<.001\right]$. The error rate in switch trials was significantly higher than in task repetition trials. The main effect of CSI was also significant $\left[F(1,30)=4.8, M S_{\mathrm{e}}=498.2, p=.036\right]$, showing the same trend revealed in the RT data (i.e., error rate decreased with long CSI). The interaction of task transition and CSI did not reach significance $[F(1,30)=2.3$, $\left.M S_{\mathrm{e}}=438.9, p=.140\right]$, and group was not significant $\left[F(1,30)=3.7, M S_{\mathrm{e}}=54.1, p=.065\right]$ and did not interact with any other variable. However, descriptively, in the go-only group the error rate was higher than in the no-go group.

Summary. After no-go trials, in which response selection was not necessary, there was no need to resolve competition between C-R rules, so residual shift cost was substantially reduced. In contrast, both no-go and go-only groups showed substantial shift costs in go trials following go trials.
When comparing overall RTs and error rates, we found that in the go-only group the error rate was higher but the RT level lower than in the no-go group. Thus, it is likely that the introduction of no-go trials slightly changed the response criterion toward slower but more accurate responses in the no-go group relative to the go-only group. However, both the RT and the error data clearly show that this did not change the general pattern of results within each group. Comparison of the go-only and no-go groups confirmed this analysis, revealing that the same effects of task preparation could be found even when some proportion of the trials did not require a response. We therefore conclude that the disappearance of residual shift cost in trials following no-go trials cannot be attributed to a change in task preparation but must indeed be due to the elimination of response-related processes.

In the no-go trials of Experiment 1, not only response selection but also the manual execution of a specific response were rendered unnecessary. Thus, our results so far do not allow us to distinguish whether the relevant process for the occurrence of residual shift cost is the selection or the execution of a response. The goal of Experiment 2 was to show that response selection rather than execution is the process that triggers the resolution of competition between $\mathrm{C}-\mathrm{R}$ rules.

\section{EXPERIMENT 2}

The motivation for running Experiment 2 was that in the no-go condition of Experiment 1, no-go trials required neither response selection nor execution (see Schuch \& Koch, 2003). Therefore, response execution might have played a role in producing the obtained pattern of no-go effects on shift cost. To test this possibility, the no-go requirement of Experiment 1 was simply replaced with the requirement to press both keys at the same time (i.e., within $50 \mathrm{msec}$ ), so that participants had to execute a motor response but were still not required to select a categoryspecific response against a competing response.

\section{Method}

Participants. Sixteen new participants were tested (11 female, 5 male; mean age $=24.75$ years) and received $€ 7$.

Stimuli, Tasks, Procedure, and Design. The experimental setting was identical to that of the no-go group of Experiment 1, with only the exception that no-go trials were replaced by "double-press" trials. The participants now reacted to a low tone by simultaneously pressing both response keys. The response on a double-press trial was classified as an error when either only one key was pressed or the two keypresses were separated by more than $50 \mathrm{msec}$. In either case, the message Bitte beide Tasten drücken ("Please press both keys") appeared on the screen.

\section{Results and Discussion}

The first two trials of each block were discarded from analysis. For RT analysis, only correct trials preceded by at least one other correct trial were included. There were $1.1 \%$ outliers (i.e., RT $>3,000 \mathrm{msec}$ ). Participants made errors in $10.3 \%$ of the go trials and $17.2 \%$ of the doublepress trials. We attribute this generally high error rate to a 
speed-accuracy tradeoff imposed by the introduction of double-press trials. Since no-go trials in Experiment 1 led participants to react more conservatively, the execution of rather fast double-press trials (see Table 2) might have led them to react more quickly but less accurately in general.

A three-way ANOVA on RT in go trials with the withinsubjects independent variables task transition (repeat vs. switch), CSI (100 vs. 1,000 msec), and previous trial type (go vs. double-press in trial $n-1$ ) revealed significant effects of task transition $\left[F(1,15)=42.3, M S_{\mathrm{e}}=7,219.2\right.$, $p<.001]$ and CSI $\left[F(1,15)=44.0, M S_{\mathrm{e}}=20,873.5, p<\right.$ $.001]$. Task-repeat trials were faster than task-switch trials, and RTs were reduced with long preparation time. The analysis also yielded a significant main effect of previous trial type $\left[F(1,15)=105.4, M S_{\mathrm{e}}=10,928.2, p<.001\right]$, indicating that RTs in go trials were slower after doublepress than after go trials. An important result, in terms of the role of response selection, was the significant interaction of task transition and previous trial type $[F(1,15)=$ 19.1, $\left.M S_{\mathrm{e}}=4,885.8, p=.001\right]$, showing that shift cost was reduced after double-press trials (from $157 \mathrm{msec}$ to $44 \mathrm{msec}$ ), even though response execution processes did occur in the double-press trials.

The interaction of task transition and CSI and the three-way interaction of task transition, CSI, and previ-

Table 2A

Experiment 2: Mean Reaction Time (in Milliseconds) of Go Trials as a Function of Task Transition (Repeat vs. Switch), Type of Trial in $\boldsymbol{n}-1$ (Go vs. Double-Press), and Cue-Stimulus Interval (CSI) in Trial $\boldsymbol{n}$ (100 vs. 1,000 msec)

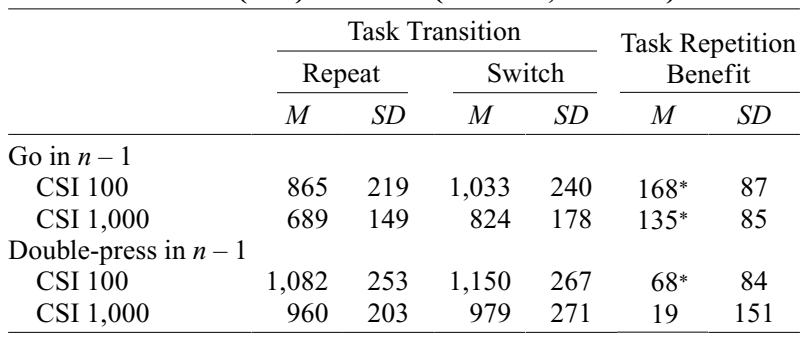

${ }^{*} p<.05$ (Task repetition benefit was tested with one-tailed $t$ tests.)

Table 2B

Experiment 2: Mean Error Percentage of Go Trials as a Function of Task Transition (Repeat vs. Switch), Type of Trial in $n-1$ (Go vs. Double-Press), and Cue-Stimulus Interval (CSI) in Trial $n$ (100 vs. $1,000 \mathrm{msec})$

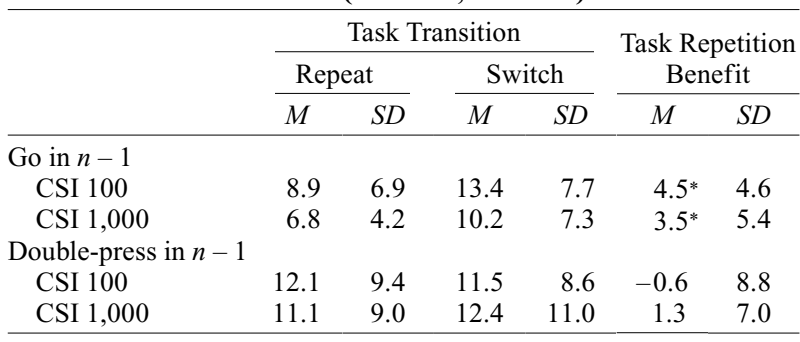

${ }^{*} p<.05$ (Task repetition benefit was tested with one-tailed $t$ tests.) ous trial type were not significant $(F<2.5)$. The interaction of previous trial type and CSI $[F(1,15)=4.7$, $\left.M S_{\mathrm{e}}=3,612.4, p=.047\right]$ was significant, indicating that the effect of a long preparation interval is stronger in go trials following a go trial $(192 \mathrm{msec})$ than in go trials following a double-press trial $(147 \mathrm{msec})$. This result suggests that task preparation was somewhat reduced after double-press trials. However, even after double-press trials there was a substantial effect of CSI.

For error rates in go trials, a three-way ANOVA was run with the within-subjects independent variables task transition, CSI, and previous trial type, revealing significant main effects of task transition $[F(1,15)=6.3$, $\left.M S_{\mathrm{e}}=23.2, p=.024\right], \operatorname{CSI}\left[F(1,15)=5.3, M S_{\mathrm{e}}=11.8\right.$, $p=.036]$, and previous trial type $\left[F(1,15)=6.9, M S_{\mathrm{e}}=\right.$ $18.1, p=.019]$. Of all interactions, only that of previous trial type and task transition was significant $[F(1,15)=$ $\left.8.1, M S_{\mathrm{e}}=13.1, p=.012\right]$, indicating a reduction of error shift cost after double-press trials (from $4.0 \%$ to $0.3 \%$ ).

In sum, the results of Experiment 2 suggest that the selection of a task-specific $C-R$ rule rather than unspecific response execution in the preceding trial is the relevant process for shift cost to occur. Like no-go trials in Experiment 1, double-press trials also strongly reduced shift cost in the subsequent trial, supporting the hypothesis that previous task-specific response selection is crucial for shift cost - that is, executing a motor response is not enough to resolve interference between C-R rules.

\section{SUMMARY AND INTEGRATION OF EXPERIMENTS 1 AND 2}

The results of Experiments 1 and 2 support the relevant role of response selection in shift cost as compared with the roles of task preparation or response execution. We can therefore be quite confident that response selection is indeed a crucial factor and that the elimination of task-specific response selection in no-go and doublepress trials is responsible for the reduction of residual shift cost. Recall that after no-go and double-press trials, the RT for a task repetition increased, whereas taskswitch trials were hardly affected by the type of the preceding trial. This result supports the idea that the difference between task-repeat and task-switch trials is more adequately described as a task repetition benefit, which is strongly reduced after no-go or double-press trials. This idea represents a relative shift in emphasis with respect to Schuch and Koch's (2003) original interpretation, which referred to a reduced shift cost. That is, we emphasize persisting activation of C-R rules to account for residual shift cost, whereas Schuch and Koch (in Experiments 2-4) focused on persisting inhibition in a three-task design. However, we do not claim that either persisting activation or inhibition alone plays a role, but that both occur and result in a persisting bias of C-R rules. Thus, both accounts are complementary and stress the role of response selection processes. The data of our experiments suggest that these processes were indeed cru- 
cially important in task switching, reinforcing Schuch and Koch's "response selection account." The data of the goonly group suggest that the effects we found are not due to generally decreased task preparation in the no-go group or to some other unintended influence introduced by using no-go trials.

Still, in interpreting the results of Experiments 1 and 2 , one might also refer to the notion that the large reduction of shift cost after no-go/double-press trials could be explained by a shift toward a "controlled" processing route instead of an "automatic" one (see, e.g., Van Duren \& Sanders, 1988). In Experiment 1, this analysis could mean that task repetitions following a go trial were processed automatically (producing a repetition benefit), whereas in every other case the controlled route had to be used, leading to an increase in RT. However, this interpretation does not contradict our account. We state that a task repetition benefit only occurs when response selection takes place in the preceding trial, resulting in an activation bias favoring a task repetition.

We could, however, think of three other alternatives to interpret the data pattern we found in Experiment 1. All three alternatives mainly concern the nature of no-go trials. First, the data pattern might be interpreted without referring to response-related processes at all, but rather by arguing that no-go trials simply constitute a different kind of task for the participants. If that were the case, either task would mean a "switch" after a no-go trial, and thus the RT should be at the level of task switches for all tasks following a no-go trial. Second, one might argue that the no-go signal leads to the inhibition of the task that was prepared during CSI. In this case, a task repetition after a no-go trial would have to overcome the selfinhibition of the task, resulting in an increased RT. Finally, a third possibility is that the general increase of RT after no-go trials might be due to priming of the no-go decision (see Schuch \& Koch, 2003). In order to test these alternative accounts, we ran a third experiment.

\section{EXPERIMENT 3}

In Experiment 3, we tested the effect of no-go trials when participants did not switch between tasks but engaged in only one numerical decision task, the magnitude or the parity task. In this single-task situation, we expected to find no sequential effects of no-go trials, because no competition between C-R rules had to be resolved for response selection. Thus, there should also be no large activation difference following go and no-go trials. However, all alternative explanations mentioned above would clearly predict substantially increased RT in go trials following no-go trials. If a no-go is coded as a different task, this should change the single-task situation into a task-switch experiment, resulting in increased RTs after no-go (resembling shift cost). If inhibition of the prepared task takes place in no-go trials, this inhibition should increase RT after no-go trials independent of the number of tasks. Finally, if there is priming of the no-go decision, it should also occur in a single-task condition. Thus, if any of these interpretations were true, we should observe a substantial RT increase after no-go trials in Experiment 3. On the other hand, the absence of such a clear increase of RT after no-go trials would favor our interpretation.

To control for the effect of the introduction of no-go trials, as in Experiment 1, half of the participants had $25 \%$ no-go trials (the no-go group), whereas the other half performed the experiment without no-go trials (the go-only group).

For comparability to Experiment 1, we also manipulated CSI. It has been argued that part of the main effect of CSI might not be task specific but rather an "unspecific" effect such as predicting target onset or becoming alert (Meiran et al., 2000), like the effect found in studies on the "foreperiod effect" (see Los \& van den Heuvel, 2001 , for a study using a choice RT task). In the single-task situation of Experiment 3, the cue has no task-specific meaning. If a main effect of CSI is observed, it would indicate that part of the CSI main effect in Experiment 1 was not task specific, because it also occurs in a situation in which no task-specific preparation is necessary. On the other hand, if there is no significant main effect of CSI, this would suggest that the effect of CSI found in Experiment 1 really reflects task-specific preparation.

\section{Method}

Participants. Sixteen new participants ( 12 female, 4 male; mean age $=24.5$ years) were tested and received $€ 5$. They were evenly assigned to the no-go and go-only groups.

Stimuli, Tasks, Procedure, and Design. Stimuli and tasks were identical to those in Experiment 1, except that each participant had to perform only one task throughout the experiment. In both groups, half of the participants performed the magnitude task, the other half the parity task. Only the task that had to be performed was mentioned to the participant. To keep the number of trials for each task equal to that in Experiment 1, only four blocks of 96 trials each were performed. The two practice blocks with short and long CSIs contained 4 (go-only group) or 6 (no-go group) trials each. For the go-only group, only CSI (100 vs. $1,000 \mathrm{msec})$ was an independent variable. For the no-go group, CSI and previous trial type (go vs. no-go in trial $n-1$ ) served as within-subjects independent variables. CSI level alternated between blocks, with CSI duration in the first block being counterbalanced across participants. As regards the analysis of go trial sequences, group (go-only vs. no-go) was a between-subjects independent variable. RTs and error rates in go trials were the dependent variables.

\section{Results and Discussion}

The first two trials of each block were discarded from analysis. The outlier criterion was applied to all trials in which the RT exceeded $1,000 \mathrm{msec}(0.7 \%$ for the goonly group and $1.6 \%$ for the no-go group). For RT analysis, only correct trials preceded by at least one other correct trial were included. The go-only group had a mean error rate of $5.1 \%$. The no-go group made errors in $1.6 \%$ of the go trials (and in $5.3 \%$ of the no-go trials).

\section{Sequential effects of no-go trials (within subjects).}

For the no-go group, a two-way ANOVA was conducted with the independent variables CSI and previous trial 
type. No effect was significant $(F \mathrm{~s}<2, p \mathrm{~s}>.2)$. If there was an RT effect at all, RT was even shorter in the short than in the long CSI condition. (The ANOVA on error rates did not obtain significant effects either, $F_{\mathrm{S}}<1$.) Most importantly, RT after no-go trials was nonsignificantly increased, by only $12 \mathrm{msec}$ (see Table 3 ).

If the general RT increase after no-go with task switching (Experiment 1) were due to a kind of "task shift," to self-inhibition of a task in no-go trials, or to priming of the no-go decision, we should have also observed a substantial effect in the present experiment. We thus conclude that no-go trials with task switching primarily eliminate shift cost that occurs after the resolution of interference in go trials, and that no sequential effects of no-go trials occur in single-task situations because there are no competing task-specific C-R rules.

Effects of the introduction of no-go trials (between subjects). We conducted a two-way ANOVA with CSI as a within-subjects variable and group as a betweensubjects variable. For this analysis, only sequences of at least two go trials (go/go) were included. The ANOVA revealed only a significant effect of the between-subjects variable group $\left[F(1,14)=11.5, M S_{\mathrm{e}}=6,247.5, p=.004\right]$, indicating that RT in the no-go group was generally higher than in the go-only group (533 vs. $438 \mathrm{msec}$ ). The effect of CSI was not significant $\left[F(1,14)=1.7, M S_{\mathrm{e}}=1,054.5\right.$, $p=.211]$, and there was no significant interaction between CSI and group $(F<1)$. Participants even tended to react slightly faster in the short than in the long CSI condition (478 vs. $483 \mathrm{msec}$ ). In fact, in the literature on foreperiod effects in single-task studies, this effect of prolonged RT with long CSI was found in studies in which CSI was manipulated blockwise (see Los \& van den Heuvel, 2001; Niemi \& Näätänen, 1981). Hence, nonspecific preparation, when isolated in Experiment 3, showed the opposite direction of the preparation effects found in Experiment 1. This indicates that the task preparation effects in Experiment 1 must have been task specific.

Error rates were subjected to the same analysis as RT data. In a two-way ANOVA with CSI and group as independent variables, the main effect of CSI was not significant and was not affected by group. The error rate was slightly lower in the long CSI condition (see Table 3 ). The main effect of group was significant $[F(1,14)=11.0$, $\left.M S_{\mathrm{e}}=9.3, p=.005\right]$, showing a higher error rate in the go-only group. That is, part of the general RT slowing due to the additional decision load in the no-go group might be related to a shift toward more accurate responses.

Summary. There was no significant increase in RT or error rate after no-go trials in Experiment 3. This result is in accordance with the response selection account. We predicted no costs after no-go trials because in a single-task experiment there is no conflict between C-R rules, and therefore no competition has to be resolved for response selection. Thus, there should be no large activation difference between go trials that follow go and no-go trials. With regard to the alternative explanations, the results of Experiment 3 suggest that participants did not experience a no-go trial as a form of switch to another "task," in which case any task following a no-go trial would have resembled a task switch. In addition, it seems unlikely that the no-go signal in Experiment 3 led participants to inhibit the task that was prepared in the CSI. It is also highly unlikely that priming of the no-go decision took place.

Finally, a comparison of the results of Experiments 1 and 3 reveals another interesting result. In the single-task condition, in which each trial is by definition a task repetition, RT in go trials that followed go trials was on average 299 msec shorter than in task-repeat trials in the task-switching experiment (go/go sequences in Experiment 1), indicating large "mixing costs" (see, e.g., Los, 1996). These mixing costs are presumably due to the need to keep active in working memory the competing alternative task (or C-R rules) in the task-switch condition (Experiment 1), which is presumably the basis for the substantial shift cost that is, according to our account, largely due to a persisting activation bias for the current $\mathrm{C}-\mathrm{R}$ rules.

\section{GENERAL DISCUSSION}

In the present study, we examined the effects of response selection on subsequent residual shift cost. We assumed that response selection leads to an activation bias favoring task repetitions. This activation bias of C-R

Table 3A

Experiment 3: Mean Reaction Time (in Milliseconds) as a

Function of Cue-Stimulus Interval (CSI) in Trial $n$ (100 vs. $1,000 \mathrm{msec}$ ), Type of Trial in $n-1$ (Go vs. No-Go), and Group (No-Go vs. Go-Only)

Trial Type in $n-1$

\begin{tabular}{|c|c|c|}
\hline Go & No-Go & No-Go Effect \\
\hline$S D$ & $M$ & $S D$ \\
\hline
\end{tabular}

No-Go Group

$\begin{array}{lllllll}\text { CSI } 100 & 528 & 58 & 545 & 47 & 17 & 26\end{array}$

$\begin{array}{lllllll}\text { CSI } 1,000 & 537 & 64 & 543 & 75 & 6 & 43\end{array}$

Go-Only Group

CSI $100 \quad 427 \quad 52$

CSI $1,000 \quad 448 \quad 66$

Table 3B

Experiment 3: Mean Error Percentage of Go Trials as a Function of Cue-Stimulus Interval (CSI) in Trial $\boldsymbol{n}(100 \mathrm{vs}$. $1,000 \mathrm{msec}$ ), Type of Trial in $n-1$ (Go vs. No-Go), and Group (No-Go vs. Go-Only)

Trial Type in $n-1$

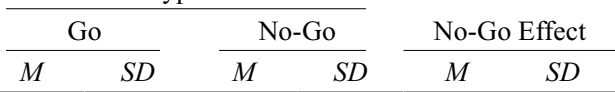

No-Go Group

$\begin{array}{lllllll}\text { CSI } 100 & 1.7 & 1.8 & 1.7 & 2.5 & 0.0 & 2.0\end{array}$

$\begin{array}{lllllll}\text { CSI } 1,000 & 1.5 & 1.8 & 1.0 & 1.4 & -0.5 & 2.2\end{array}$

Go-Only Group

CSI $100 \quad 5.3 \quad 3.6$

CSI $1,000 \quad 5.0 \quad 1.8$


rules could account for a large part of so-called residual shift cost. To study the role of response selection, we used a go/no-go methodology. In Experiment 1, converting a portion of the trials of a task-switching experiment into no-go trials rendered response selection unnecessary in these trials. The results indicated that a large part of shift cost is due to response selection (and not response execution, as we determined in Experiment 2) in the preceding trial. Comparison of the results of the no-go and go-only groups suggested that the introduction of no-go trials did not impair preparation processes in the no-go group. In addition, data from a single-task experiment (Experiment 3 ) strongly suggested that the effect of no-go trials indeed results from the elimination of response selection rather than from no-go trials being coded as an additional task or from inhibition of the prepared task in no-go trials. Taken together, the present experiments contribute major findings concerning task preparation, the resolution of interference, and the nature of no-go trials.

\section{Task Preparation}

Although we aimed at studying the role of responserelated processes, we do not wish to deny that task preparation is important in task switching (e.g., Meiran et al., 2000). The preparation-based reduction of shift cost is mainly caused by a reduction of the task-switch RT. We assume that this happens because in the task-specific preparation for the current trial, the relevant task set, including stimulus categories, is activated. Since in taskrepeat trials the relevant task is already highly activated, in those trials only a small effect of preparation can be found. In switch trials, the preparatory activation of the currently relevant task changes the unfavorable activation status of this task, leading to a reduction in RT. However, it is important to note that these effects may be rather "fragile" and can presumably be dominated by the effects of specific response selection processes (see Kleinsorge, Gajewski, \& Heuer, 2005). Therefore, it is important to distinguish between the effects of task preparation and response selection in task switching.

\section{Response Selection and the Task \\ Repetition Benefit}

To explain the role of response selection in shift cost, we assume that for response selection to take place, competition between C-R rules has to be resolved. Relevant $\mathrm{C}-\mathrm{R}$ rules are strongly activated and, at the same time, currently irrelevant competing rules are presumably inhibited (see Schuch \& Koch, 2003). Thus, after response selection, the activation level of C-R rules is different for relevant and irrelevant rules. As the difference of activation persists, at least to some degree, into the next trial, there is an advantage of repeating the same task.

The present data suggest a slight theoretical modification of the suggestion made by Schuch and Koch (2003). We observed a strong increase in RT for task repetitions after no-go trials as compared with those after go trials, whereas only very little change in RT occurred for task switches. A no-go in trial $n-1$ thus eliminated the benefit of repeating the same task, but it did not facilitate task-switch trials. Thus, we propose that the activation difference between $\mathrm{C}-\mathrm{R}$ rules existing after response selection seems to facilitate a task repetition in the following trial more than it impedes a task switch. In contrast, Schuch and Koch assumed an additional process of no-go decision priming, accounting for a general increase in RT after every no-go trial. Although this appeared reasonable, our single-task Experiment 3 showed no evidence for this no-go priming, since no general RT increase occurred in go trials following no-go trials. Therefore, we believe that reduction of a task repetition benefit after no-go trials is an appropriate explanation of the present data pattern for participants switching between two tasks (see also, e.g., Allport \& Wylie, 1999; Dreisbach, Haider, \& Kluwe, 2002; Sohn \& Carlson, 2000).

Our account of the role of response selection in task switching can be related to Allport et al.'s (1994) notion of "task set inertia," because persisting activation (and inhibition) produce residual shift cost, and we can specify that this kind of "proactive interference" is largely caused by response selection processes. We also believe that this role of response selection can account for the fact that task preparation is usually incomplete, in line with Rogers and Monsell's (1995) proposed idea of "stimulus-cued completion" of task set reconfiguration, but our results show that it is not the stimulus but rather the stimulus-based response selection process that "completes" the task preparation. Our account also appears consistent with Meiran's (2000) suggestion of a process of "response recoding." Here, we have shown that response selection indeed biases C-R rules in favor of the task in the current trial, and that this bias persists into the next trial.

\section{The Effect of No-Go Trials}

A major empirical contribution of our study is that we ruled out several alternative explanations concerning the sequential effects of no-go trials by showing that such trials are not coded as a different task, that there is no self-inhibition of the task in a no-go trial, and that there is no priming of the no-go decision. Still, some questions need further discussion.

For example, it might be argued that the single-task situation of Experiment 3 was not suitable for drawing conclusions on the nature of no-go effects in more complex settings, such as task-switching experiments, because no-go effects might depend on the experimental paradigm. In single-task experiments, a task might not be inhibited in no-go trials because there is only one relevant task. In contrast, in a task-switching experiment, the no-go condition could lead to self-inhibition of a task in order to ensure equal preparation for each task in the following trial. However, explaining no-go effects in taskswitching experiments by the self-inhibition of a task bears the problem of accounting for the effects of no-go trials on the backward-inhibition effect (see Schuch \& Koch, 2003). In a task-switching experiment with three 
tasks, the $n-2$ repetition cost (i.e., the higher RT and error rate in the last trial of an $\mathrm{ABA}$ rather than a CBA task sequence) was strongly reduced after a no-go trial. The self-inhibition of the task in trial $n-1$ (i.e., task B in the sequences described above) could not account for the difference between a task repetition and a task switch from trial $n-2$ to trial $n$. The response selection account, on the other hand, can easily explain this effect in terms of the missing resolution of interference in no-go trials (for a more detailed explanation, see Schuch \& Koch, 2003). Thus, we prefer to rely on one mechanism that is able to explain all phenomena rather than to assume different mechanisms for single-, two-, and three-task experiments.

We assume that response selection is the mechanism that induces an activation bias favorable for the currently relevant C-R rules. This bias gets weaker over time, so that it needs to be "refreshed" in each trial in order to obtain the relative benefit of repeating rather than switching tasks in a situation with mixed tasks. Our data suggest that this weakening of the bias proceeds relatively quickly, so that the influence of this bias is no longer measurable when the intervening trial was a no-go (or double-press) trial. In other words, the data suggest that a major part of the proactive interference of $\mathrm{C}-\mathrm{R}$ rules is short-lived. Also, since there are no competing $\mathrm{C}-\mathrm{R}$ rules in a single-task situation, we do not find such a strong influence of no-go trials on the subsequent performance in go trials in this context.

Underlying our experiments (and most notably Experiment 1) is the assumption that in no-go trials, taskspecific preparation takes place but response selection is rendered unnecessary. However, it seems very difficult to prove, in a strict sense, that our participants indeed did not select a $\mathrm{C}-\mathrm{R}$ rule. The presentation of the stimulus, although it could be ignored in no-go trials, might have triggered the selection of a C-R rule automatically. We do not deny that participants are likely to categorize the stimulus even in no-go trials, but we presently assume that no application of a C-R rule takes place.

The data of Experiment 3 suggest that a prepared task is not inhibited in a no-go trial. However, it might still be argued that no-go trials include the active suppression of a motor response (see Band \& van Boxtel, 1999, for a review). Thus, one important question is whether the no-go response (i.e., no reaction) is a result of motor inhibition of the response that would have been correct in a go trial. This question is related to that of whether response selection takes place in no-go trials, since a motor response first needs to be selected before it can be inhibited.

Because there is no overt response in a no-go trial, studies examining the effects of a no-go condition with EEGs are of interest in the present context. In an EEG, a no-go trial usually goes along with an inhibitory change in the signal, the so-called NoGo-N2, but it is not clear whether this inhibition takes place at the motor level (Falkenstein, Hoormann, \& Hohnsbein, 1999). In most EEG studies, a simple go/no-go decision has been used. Thus, in task preparation, a motor response could have been prepared, which then had to be inhibited in a no-go trial. In our experiments, during task preparation only the abstract task set (or set of C-R rules), but not a motor response, could be prepared, because the correct response could be chosen only after the presentation of the go/no-go signal and stimulus. Regarding this difference in designs (i.e., simple response vs. choice response), a recent study by Smid, Fiedler, and Heinze (2000) has suggested that the inhibition that takes place in no-go trials indeed depends on the complexity of the task. The authors compared no-go trials in a simple go/no-go task and in a choice go/no-go task (one with two different go responses but one no-go), and found that a no-go N200 was more negative in the simple than in the choice go/no-go task. Thus, in the simple go/no-go task, some indication was found of the inhibition of a specific response, which was not found when there was more than one overt response possible. However, in Smid et al.'s study, no-go trials did not replace a possible response in go trials but were a third alternative. Still, the results of this study show that findings from simple go/no-go tasks indicating motor inhibition of a prepared response cannot easily be generalized to more complex settings. Nevertheless, it would be interesting to complement our present no-go approach with EEG methods in order to examine further the functional locus of the assumed activation bias of $\mathrm{C}-\mathrm{R}$ rules.

Note also that in our present account, the activation bias responsible for the task repetition benefit is assumed to be a consequence of response selection processes but that, strictly speaking, a role of even "later" response processes cannot be excluded. The data of our Experiment 2 (double-press instead of no-go trials) suggest that the lack of any response execution does not prevent an activation bias from developing and persisting into the next trial. However, it might well be that processes of monitoring and evaluating a task-specific response, once selected and initiated, play important but still unexplored roles, as is assumed in conflict monitoring models (Botvinick, Braver, Barch, Carter, \& Cohen, 2001). It remains for future research to explore whether processes that occur even later in the processing chain (possibly even postresponse) may contribute to shift cost.

\section{Conclusion}

We propose that a large share of the so-called "residual" shift cost in task switching is due to effects of response selection in the preceding trial. We assume that response selection resolves competition between C-R rules, producing a strong benefit for a task repetition. When response selection does not take place (i.e., in no-go trials), this benefit is abolished. Although cuebased task preparation more strongly affects task-switch trials (i.e., by reducing shift cost), response selection processes seem to be primarily responsible for the "residual" part of shift cost, which actually is better termed a task repetition benefit. 


\section{REFERENCES}

Allport, D. A., Styles, E. A., \& Hsieh, S. (1994). Shifting intentional set: Exploring the dynamic control of tasks. In C. Umiltà \& M. Moscovitch (Eds.), Attention and performance $X V$ : Conscious and nonconscious information processing (pp. 421-452). Cambridge, MA: MIT Press, Bradford Books.

Allport, [D.] A., \& Wylie, G. (1999). Task-switching: Positive and negative priming of task-set. In G. W. Humphreys, J. Duncan, \& A. M. Treisman (Eds.), Attention, space, and action: Studies in cognitive neuroscience (pp. 273-296). Oxford: Oxford University Press.

BAND, G. P. H., \& VAN Boxtel, G. J. M. (1999). Inhibitory motor control in stop paradigms: Review and reinterpretation of neural mechanisms. Acta Psychologica, 101, 179-211.

Botvinick, M. M., Braver, T. S., BarCh, D. M., Carter, C. S., \& Cohen, J. D. (2001). Conflict monitoring and cognitive control. Psychological Review, 108, 624-652.

DE JONG, R. (2000). An intention-activation account of residual switch costs. In S. Monsell \& J. Driver (Eds.), Control of cognitive processes: Attention and performance XVIII (pp. 357-376). Cambridge, MA: MIT Press, Bradford Books.

Dreisbach, G., Haider, H., \& Kluwe, R. H. (2002). Preparatory processes in the task-switching paradigm: Evidence from the use of probability cues. Journal of Experimental Psychology: Learning, Memory, \& Cognition, 28, 468-483.

FaLKenstein, M., HoormanN, J., \& Hohnsbein, J. (1999). ERP components in go/nogo tasks and their relation to inhibition. Acta Psychologica, 101, 267-291.

HoffmanN, J., Kiesel, A., \& Sebald, A. (2003). Task switches under go/nogo conditions and the decomposition of switch costs. European Journal of Cognitive Psychology, 15, 101-128.

JERSILD, A. T. (1927). Mental set and shift. Archives of Psychology (Whole No. 89).

Kleinsorge, T., Gajewski, P. D., \& Heuer, H. (2005). Task sets under reconstruction: Effects of partially incorrect precues. Quarterly Journal of Experimental Psychology, 58A, 521-546.

КосH, I. (2003). The role of external cues for endogenous advance reconfiguration in task switching. Psychonomic Bulletin \& Review, 10, 488-492.

Los, S. A. (1996). On the origin of mixing costs: Exploring information processing in pure and mixed blocks of trials. Acta Psychologica, 94, 145-188.

Los, S. A., \& van DEN Heuvel, C. E. (2001). Intentional and unintentional contributions to nonspecific preparation during reaction time foreperiods. Journal of Experimental Psychology: Human Perception \& Performance, 27, 370-386.

MAYR, U., \& KEELE, S. W. (2000). Changing internal constraints on action: The role of backward inhibition. Journal of Experimental Psychology: General, 129, 4-26.

MEIRAN, N. (1996). Reconfiguration of processing mode prior to task performance. Journal of Experimental Psychology: Learning, Memory, \& Cognition, 22, 1423-1442.

MEIRAN, N. (2000). Modeling cognitive control in task-switching. Psychological Research, 63, 234-249.

Meiran, N., Chorev, Z., \& SAPIR, A. (2000). Component processes in task switching. Cognitive Psychology, 41, 211-253.

Niemi, P., \& NÄ̈̈TÄNEN, R. (1981). Foreperiod and simple reaction time. Psychological Bulletin, 89, 133-162.

Rogers, R. D., \& MonSELL, S. (1995). Costs of a predictable switch between simple cognitive tasks. Journal of Experimental Psychology: General, 124, 207-231.

RubinSTEIN, J. S., MEYER D. E., \& Evans, J. E. (2001). Executive control of cognitive processes in task switching. Journal of Experimental Psychology: Human Perception \& Performance, 27, 763-797.

ScHUCH, S., \& KocH, I. (2003). The role of response selection for inhibition of task sets in task shifting. Journal of Experimental Psychology: Human Perception \& Performance, 29, 92-105.

SMID, H. G. O. M., FiedLeR, R., \& HeinZe, H.-J. (2000). An electrophysiological study of the insertion of overt response choice. Journal of Experimental Psychology: Human Perception \& Performance, 26, 1053-1071.

SoHN, M.-H., \& CARLSON, R. A. (2000). Effects of repetition and foreknowledge in task-set reconfiguration. Journal of Experimental Psychology: Learning, Memory, \& Cognition, 26, 1445-1460.

VAN DuREN, L. L., \& SANDERS, A. F. (1988). On the robustness of the additive factors stage structure in blocked and mixed choice reaction designs. Acta Psychologica, 69, 83-94.

(Manuscript received March 7, 2003; revision accepted for publication August 15, 2004.) 